\title{
Corrigendum: Electroacupuncture Combined With Diet Treatment Has a Therapeutic Effect on Perimenopausal Patients With Abdominal Obesity by Improving the Community Structure of Intestinal Flora
}

\begin{abstract}
Jili Sheng ${ }^{1 \dagger}$, Geyao Yang ${ }^{2 \dagger}$, Xiaoqing Jin ${ }^{1}$, Caijuan $\mathrm{Si}^{3}$, Yuan'an Huang ${ }^{4}$, Zhouxiao Luo ${ }^{5}$, Tao Liu ${ }^{1}$ and Jianfang Zhu ${ }^{1 *}$

${ }^{1}$ Acupuncture Department, Zhejiang Hospital, Hangzhou, China, ${ }^{2}$ Acupuncture and Massage Department, Hangzhou Geriatric Hospital, Hangzhou, China, ${ }^{3}$ Nutritional Department, Zhejiang Hospital, Hangzhou, China, ${ }^{4}$ Massage Department, Zhejiang Hospital, Hangzhou, China, ${ }^{5}$ Acupuncture Department, Tonglu TCM Hospital, Hangzhou, China
\end{abstract}

Keywords: abdominal obesity, perimenopausal syndrome, 16S rRNA, intestinal flora, electroacupuncture

\section{A Corrigendum on}

Electroacupuncture Combined With Diet Treatment Has a Therapeutic Effect on Perimenopausal Patients With Abdominal Obesity by Improving the Community Structure of Intestinal Flora

by Sheng, J., Yang, G., Jin, X., Si, C., Huang, Y., Luo, Z., Liu, T., and Zhu, J. (2021). Front. Physiol. 12:708588. doi: 10.3389/fphys.2021.708588

In the original article, there was an error in the Ethics Statement as published. A correction has been made to Materials and Methods subsection Ethics Statement and to the Ethics Statement:

"The study has been approved by the Ethics Committee of Zhejiang Hospital [No. 2018 (9K)] and all patients signed the written informed consent."

The authors apologize for this error and state that this does not change the scientific conclusions of the article in any way. The original article has been updated.

Publisher's Note: All claims expressed in this article are solely those of the authors and do not necessarily represent those of their affiliated organizations, or those of the publisher, the editors and the reviewers. Any product that may be evaluated in this article, or claim that may be made by its manufacturer, is not guaranteed or endorsed by the publisher.

Copyright (c) 2022 Sheng, Yang, Jin, Si, Huang, Luo, Liu and Zhu. This is an open-access article distributed under the terms of the Creative Commons Attribution License (CC BY). The use, distribution or reproduction in other forums is permitted, provided the original author(s) and the copyright owner(s) are credited and that the original publication in this journal is cited, in accordance with accepted academic practice. No use, distribution or reproduction is permitted which does not comply with these terms. 